\title{
AN ANALYSIS OF THE AIR-JET YARN-TEXTURING PROCESS PART V: THE EFFECT OF WETTING THE YARNS
}

\author{
M. ACAR, R. K. TURTON, and G. R. WRAY \\ Loughborough University, UK
}

\begin{abstract}
It is shown that the quantity of water mixing into the air-flow is an insignificant proportion of the total amount of water used in the air-jet texturing process and that this has a negligible effect on the air-flow in the texturing nozzle. It is suggested that only a fraction of this water is needed to impart the desired effects of wetting. Experimental investigations show that water acts as a lubricant to reduce the filament-filament and filamentsolid-surface friction and hence aids the longitudinal displacements of the filaments relative to each other. A realignment of the yarn path minimizes the friction between the filaments and solid surfaces.
\end{abstract}

\section{INTRODUCTION}

Modern industrial practice often involves wetting of the yarns during the air-jet texturing process by passing the supply yarn through a water bath, wetting head, or spray unit, either integrated with the texturing nozzle or as a separate wetting system as shown in Fig. 1 of Part IV ${ }^{1}$. Regardless of the application technique, wetting of the yarns results in improved process stability (see Part III²) and consequently in better yarns (see Part VII ${ }^{3}$ ). It should be noted that, although wetting improves texturing, it may cause the spin finish applied to the supply yarn by the fibre manufacturers, and any impurities in the water, to contaminate the nozzle. This reduces the nozzle efficiency and can cause stoppages of the process for cleaning the contaminated nozzles.The use of water may also result in undesirably high moisture content in the final textured yarn.

\section{PREVIOUS WORK}

Some publications acknowledge the importance of wetting the yarn during the air-jet texturing process to obtain improved yarn quality but fail to provide satisfactory explanations of how such wetting improves both the effectiveness of texturing and the resultant yarn quality. Fischer ${ }^{4}$,although offering no experimental evidence to support his hypothesis, claimed that the effect of wetting as a means of reducing the inter-fibre friction was limited. By referring to earlier investigations into flow conditions in supersonic nozzles, which had demonstrated that humidity increases the strength of so-called 'condensation shocks', he postulated that filaments are deflected towards the jet axis after such a condensation shock, a phenomenon that he claimed 
might assist the 'interlacing' of the filaments. Later, Bock and Luenenschloss ${ }^{5}$ surmised that wetting the filaments could alter the flow and thereby improve the yarn quality. They also referred to possible condensation effects that the damp air could cause, but, like Fischer ${ }^{4}$, they offered no experimental evidence.

\section{EFFECTS OF WATER ON THE AIR-FLOW}

Yarns passing through the wetting head carry entrained water until they reach the nozzle, where the secondary air-flow causes a substantial amount of water to be sprayed away so that only a fraction of the total quantity of the water used is dragged along with the filaments into the nozzle. Most of this water, when it meets the incoming jets from the inlet bores at sonic speeds, will be blown off the filaments and broken down into very small mist droplets, which could be blown out with the primary flow. These water particles will presumably have some effect on the primary flow.

The effect of entrained water on the flow properties can be estimated by considering the flow of a gas-particle mixture. Homogeneous-equilibrium-flow theory gives the properties of a two-phase mixture containing particles in terms of the properties of the air and particle components in the flow. Since the water is mixed into the air in the form of a mist, it can be assumed that this two-phase (gas-particle-mixture) flow consists of air and homogeneously suspended water particles. Hence, homogeneousequilibrium-flow theory can be applied to the air-particle mixture in the texturing nozzles, and the effects of water on the flow properties can be calculated.

This approach was applied by the authors to converging-diverging-type texturing nozzles. A nozzle with a throat diameter of $2 \mathrm{~mm}$ and a diverging section that gives a complete expansion was assumed for the calculations. The flow velocities at the exit plane of the nozzles for air only and for air-water mixtures are compared in Table I for various operating pressures; for the same working pressures, the water-tocompressed-air consumption rates (expressed as percentages) are also given.

These theoretical calculations showed that wetting the yarn has only a very slight effect on the flow exit velocity, depending on the amount of water mixed into the air. Wetting may tend to produce a reduction in flow velocity, which in turn would have an adverse effect on the texturing process.

Table 1

Comparison of Free-air-flow Velocities with Those of Flows of Air-Water Mixtures for Varying Pressure and Varying Water Contents in the Air-flow for Converging-Diverging Nozzle

\begin{tabular}{lccccc}
\hline & & & \multicolumn{3}{c}{ Axial Velocity at the Exit Plane $(\mathrm{m} / \mathrm{s})^{*}$} \\
\cline { 4 - 6 } $\begin{array}{l}\text { Working } \\
\text { Pressure } \\
(\mathrm{MPa})\end{array}$ & $\begin{array}{c}\text { Air-mass } \\
\text { Flow-rate } \\
(\mathrm{kg} / \mathrm{h})\end{array}$ & Air only & $\begin{array}{c}0.2 \mathrm{~L} / \mathrm{h} \\
\text { Water }\end{array}$ & $\begin{array}{c}0.5 \mathrm{~L} / \mathrm{h} \\
\text { Water }\end{array}$ & $\begin{array}{c}1.0 \mathrm{~L} / \mathrm{h} \\
\text { Water }\end{array}$ \\
\hline 1.0 & 16.3 & 529 & $527(1.2)$ & $525(3.1)$ & $521(6.1)$ \\
0.9 & 14.7 & 520 & $518(1.4)$ & $516(3.4)$ & $511(6.8)$ \\
0.8 & 13.1 & 510 & $508(1.5)$ & $505(3.8)$ & $499(7.7)$ \\
0.7 & 11.4 & 497 & $495(1.8)$ & $491(4.4)$ & $485(8.8)$ \\
0.6 & 9.8 & 482 & $479(2.0)$ & $475(5.1)$ & $466(10.2)$ \\
0.5 & 8.2 & 462 & $458(2.5)$ & $453(6.1)$ & $445(12.3)$ \\
0.4 & 6.5 & 435 & $430(3.1)$ & $424(7.7)$ & $413(15.3)$ \\
\hline
\end{tabular}

${ }^{*}$ Figures in brackets show the mass flow-rate of water expressed as a percentage of the mass flow-rate of air. 


\section{SIGNIFICANCE OF THE QUANTITY OF WATER USED}

Bock and Luenenschloss ${ }^{5}$ applied water to their supply yarns over the range from 0 to $1.5 \mathrm{~L} / \mathrm{h}$, but only when reduced to $0.1 \mathrm{~L} / \mathrm{h}$ did it cause significant decreases in the process stability and the number ofloops in the textured yarn. Water-consumption rates higher than this do not affect the properties of textured yarns. Similarly, Artunc's work ${ }^{6}$ on the moisture content of the textured yarns showed that the quantity of water is critical only at consumption rates of less than $0.2 \mathrm{~L} / \mathrm{h}$. It therefore appears that only a small amount of water is needed to have an effect on the texturing process and. hence on the resultant yarn properties.

A simple experiment was devised to observe the effects of the quantity of water used during the process whereby the yarn tension in the feed zone (see also Section 7) was measured while the water-consumption rate was gradually increased.It was observed that, as soon as the water was turned on (approximately $0.2 \mathrm{~L} / \mathrm{h}$ was the minimum measurable amount), the tension that occurred in the immediately previous dry-texturing state increased to the level of the tension that was previously observed in wet texturing and that further increases in the water-consumption rates had no effect on this tension. This provided further evidence that only a small amount of water is required to impart the desired effects of wet texturing.

\section{QUANTITY OF THE WATER ENTRAINED INTO THE PRIMARY AIR STREAM}

To quantify the amount of water entrained into the nozzle, the conventional singlechamber nozzle-enclosure unit was modified by providing a sealed central vertical wall to divide it into two separate chambers, the nozzle housing being fitted into a cutout in this separating wall. Thus the water blown away by the secondary flow was kept in the chamber adjacent to the nozzle entry region \Secondary-flow chamber), while the water that was entrained into the primary flow was collected in that embracing the nozzle exit region (primary-flow chamber).

The intention was to collect the water separately from both chambers and determine their respective ratios to the total amount of water used, which was measured by a rotameter. However, it was observed that no measurable amount of entrained water was collected in the primary-flow chamber, which remained clear throughout the process, whereas the secondary-flow chamber was filled with misty air, which naturally condensed into collectable water. It can be concluded that any water entrained within the primary air-flow is insignificant and therefore that any effects that this might have on the flow itself will be negligible. Hence the postulations of Fischer ${ }^{4}$ and of Bock and Luenenschloss ${ }^{5}$, which in essence claim that 'wetting affects the air-flow', are almost completely invalid. Consequently, the significant improvements observed in the proper- ties of wet-textured yarns, to be

described in Part $\mathrm{VII}^{3}$, could be due to a reduction in friction, as will be discussed in in this paper.

\section{FRICTION IN THE AIR-JET TEXTURING PROCESS}

The frictional behaviour of lubricated textile fibres and yarns passing over surfaces is dominated by hydrodynamic factors, and Fig. 1 shows the behaviour to be 
expected from textile fibres and yarns making contact with solid surfaces 7,8 . Schick, in a series of papers ${ }^{9-12}$, showed that the friction in the hydrodynamic region increases with increasing yarn speed, lubricant viscosity, surface pressure, and area of contact. He also showed that surface roughness plays an important role in frictional behaviour and that this is independent of whether the yarn or the contacting surface is contributing to the particular value of surface roughness. Schick further concluded that there is apparently no significant difference between the frictional behaviour of fibres contacting solid surfaces and that of fibres contacting other fibres.

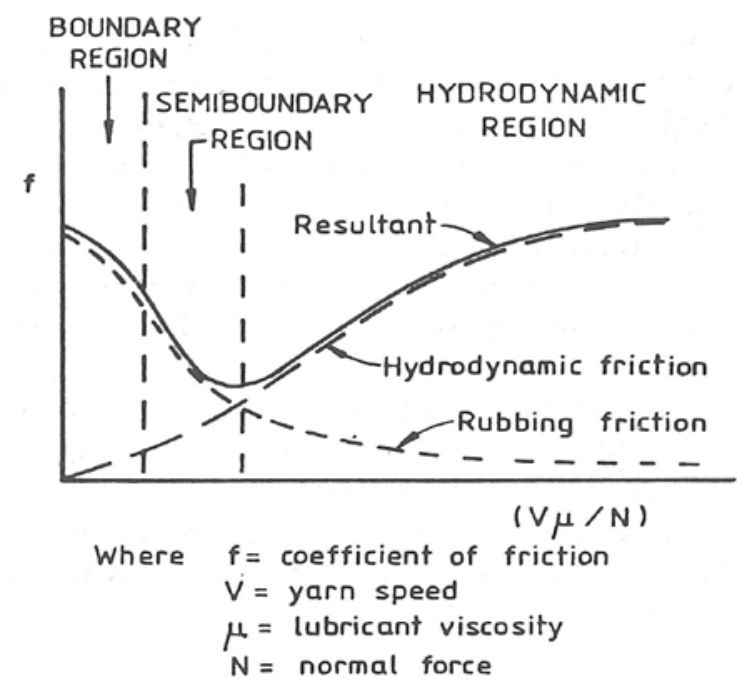

Fig. 1. Typical friction behaviour of textile fibres and yarns (after Hansen and Tabor ${ }^{7}$ and Olsen ${ }^{8}$ )

The effect of friction in the air-jet texturing process can be investigated in the light of Schick's findings. In the texturing zone, filaments make contacts with the internal surface of the wetting unit and with the yarn guides preceding the nozzle before they make further contacts with the nozzle's internal surface. As Wray ${ }^{13}$ showed, longitudinal displacements of the filaments occur relative to each other (see Part $\mathrm{III}^{2}$ ) and these cause the filaments to rub together. Both fibresolid-surface friction and fibre-fibre friction therefore occur during the air-jet texturing process, and the relative velocities are such that these are well within the hydrodynamic range. Friction between the yarns and the contacting surfaces will oppose the motion of the filaments along their path, whereas inter-filament friction will oppose their longitudinal displacements during texturing.

The fluid forces, which blow the filaments out of the nozzle, create a tension in the segment of the filaments in the feed zone, i.e., between the feed rollers and the nozzle. The resultant force acting on the filaments will be determined by the fluid forces and the opposing frictional forces. If the fluid forces are kept constant, any changes that may occur in the continuous-filament-yarn tension within this feed-zone can be taken as an indication of changes in the frictional forces; hence the effects of wetting the filaments on the frictional forces during the air-jet texturing process can be analysed by such feed-zone tension measurements. 


\section{EFFECTS OF WETTING ON FRICTION IN AIR-JET TEXTURING}

Fig. 2 shows the results of experiments conducted with the conventional texturing arrangement of the nozzle and the yarn-feed system (see Fig. 1 of Part IV $^{1}$ ). The supply yarn (175-dtex /66-fil single-end polyester-fibre yarn) specified in Part III ${ }^{2}$ was guided through the wetting unit and passed over a yarn guide prior to its entry to the nozzle, where further yarn-surface contacts occurred, all of which would cause yarn-solid-surface friction. A water-consumption rate of $1.0 \mathrm{~L} / \mathrm{h}$ was used.
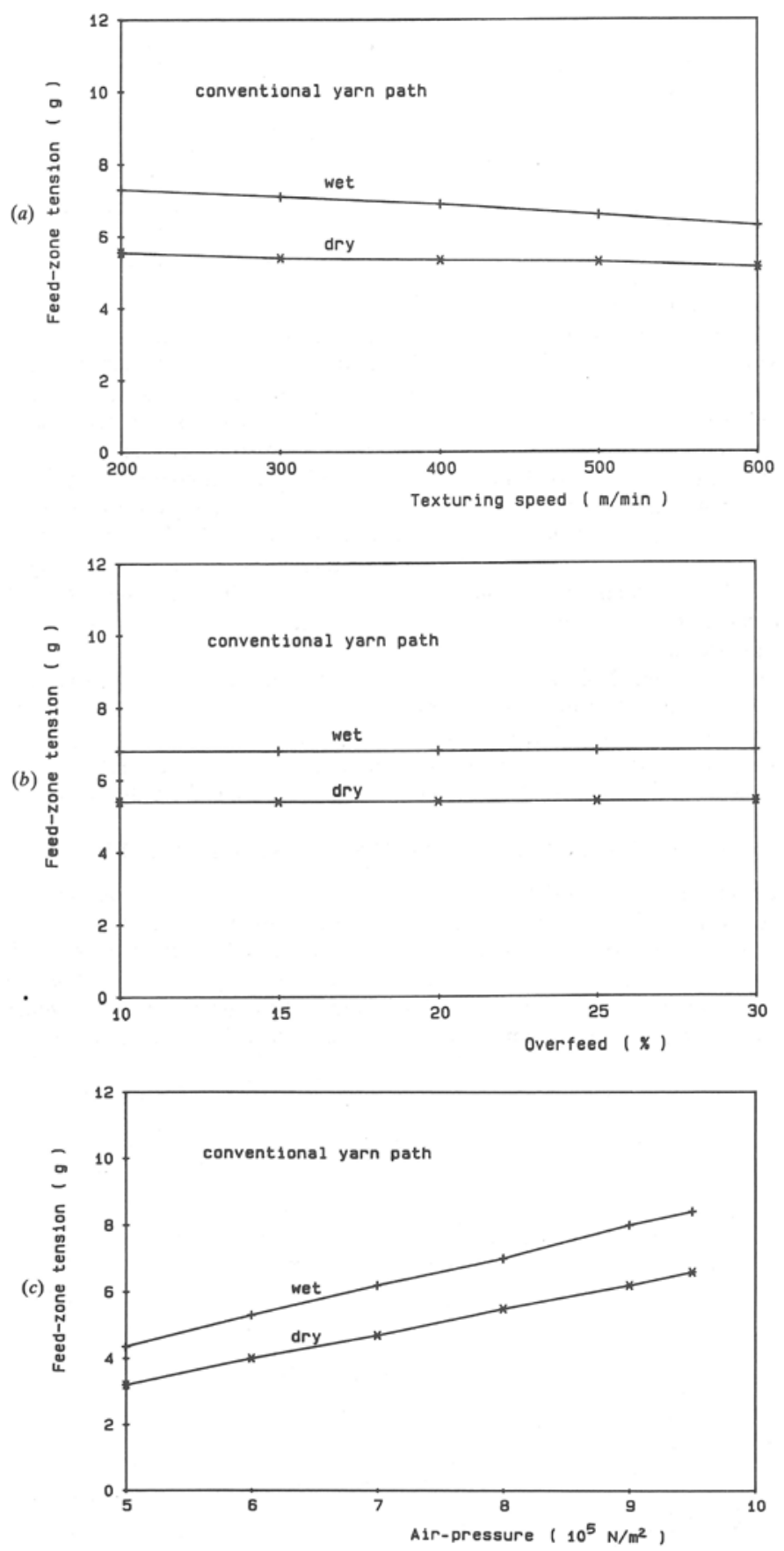

Fig. 2. Variation of tension in the feed zone with the conventional yarn path for changes in: (a) texturing speed; (b) overfeed ratio; and (c) air-pressure 
The effects of varying texturing speed, overfeed, and air-pressure can be seen from Fig. 2(a), (b), and (c), respectively, for both dry- and wet-texturing conditions. Whether dry to wet, the rate of increase in the friction with increasing texturing speed was observed to be small, as indicated in Fig. 2(a) by the small decreases in the feed-zone tension. This is in agreement with the general frictional behaviour of textile fibres and yams, which varies little with speed in this processing-speed range ${ }^{9}$ Fig. 2(b) shows no significant change in the tensions with increasing values of overfeed because changing this does not affect the parameters that determine the frictional behaviour of and Fig. 2(c) shows an approximately linear relation for both dry and wet texturing. This increase is due to the increased fluid-drag forces acting on the filaments at the higher airpressures.

In general, Fig. 2 shows that the tensions in the yam when wet-textured are substantially higher than those under dry-texturing conditions, which thereby indicates a reduction in the friction.The experimental and theoretical evidence suggests that this is caused by the lubrication effects of the water and is not a consequence of the changes in flow properties as suggested by Fischer ${ }^{4}$ and by Bock and Luenenschloss ${ }^{5}$.

\section{REALIGNMENT OF THE YARN PATH TO REDUCE FRICTION}

Experiments were also devised to investigate the effects of a reduction in friction between the yam and the contacting solid surfaces. As shown in Fig. 3, the feed rollers, wetting unit, and texturing nozzle were realigned to reduce their contact with the supply yam, and the yam guide preceding the nozzle was removed so that the yam assumed a straight path between the feed rollers and the nozzle.

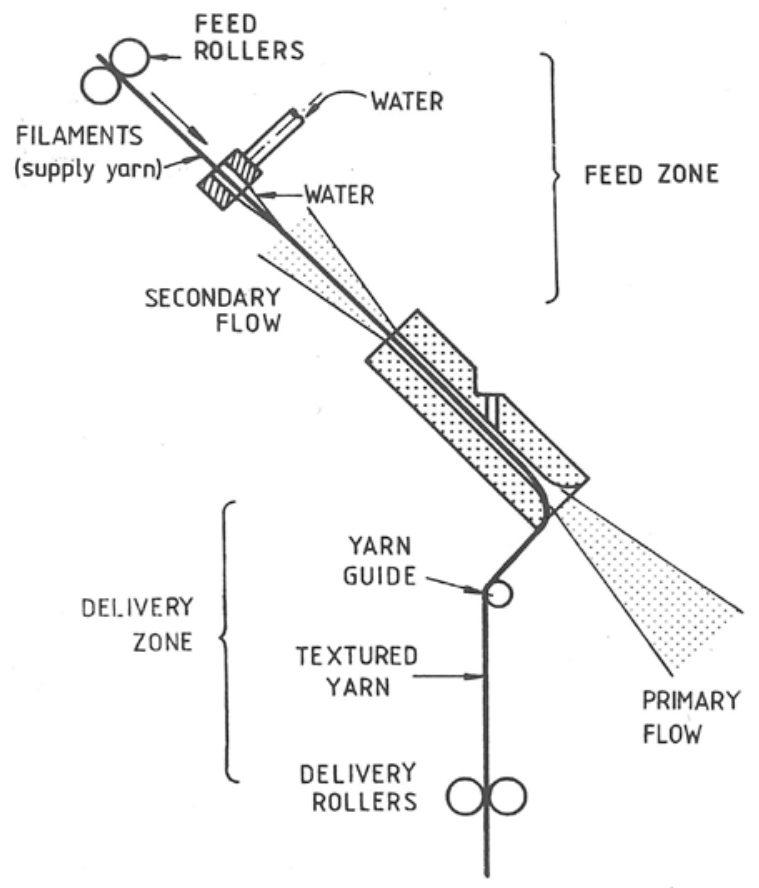

Fig. 3. Realigned texturing arrangement to reduce friction between the yarn and 
the contact surfaces

On emergence from the nozzle, it was forced to deflect at right angles, which is known to be an essential requirement for effective texturing, by the use of an additional yam guide. Since this yam guide is situated after the nozzle, it will have no effect on the tension in the yam between the feed rollers and the nozzle.

The results of the feed-zone tension measurements with this realigned yarn path are given in Fig. 4(a), (b), and (c) for varying texturing speed, overfeed ratio, and air-pressure, respectively. These show that the tensions for both dry and wet texturing with the new arrangement are higher than the corresponding tensions obtained with the conventional texturing arrangement and suggest that the reduced frictional contact in the new arrangement is a factor causing increased tension before the jet.

\section{EFFECTS OF WETTING ON INTER-FILAMENT FRICTION}

With the realigned yarn path, there are only small differences between the tensions in the feed zone for wet and dry texturing, which thus suggests that similar forces act on the filaments in both cases. This may be an indication of similar texturing conditions, and it may therefore be argued that the production of yarns with similar characteristics may be expected from both dry and wet processing. However, in Part III', the present authors have shown that tension in the yarn in the delivery zone, i.e., between the nozzle and the delivery rollers, is an indication of the effectiveness of texturing. They also argue that the tension in the stabilizing zone, i.e., between the delivery rollers and the take-up rollers, gives a comparative measure of the loop stability. Figures 5, 6, and 7 show the yarn tensions in the delivery and stabilizing zones with the realigned yarn path for varying texturing speed, overfeed, and air-pressure, respectively. These results show substantial differences in delivery- and stabilizing-zone tensions for dry and wet texturing conditions, indicating a better texturing effect and loop stability from wet texturing, despite the similar external fluid and frictional forces acting on the filaments in both dry and wet texturing conditions.

Although the frictional forces between the filaments and the contacting surfaces in dry texturing with the realigned yarn path are reduced owing to there being fewer solid contacts, the inter-filament friction should remain at the levels corresponding to those occurring with the conventional yarn path because the realigned yarn path would not be expected to alter the inter-filament contacts significantly. This high friction between the filaments in dry texturing will resist their longitudinal displacements relative to each other and thereby result in an inferior texturing performance. Since wetting reduces the inter-filament friction and makes for easier longitudinal displacement of the filaments with respect to each other, this, in turn, encourages the formation of loops, which then become entangled as they emerge from the nozzle, as will be discussed more fully in Part VI ${ }^{14}$. By means of this mechanism, the wetting of the filaments enhances the texturing process. 

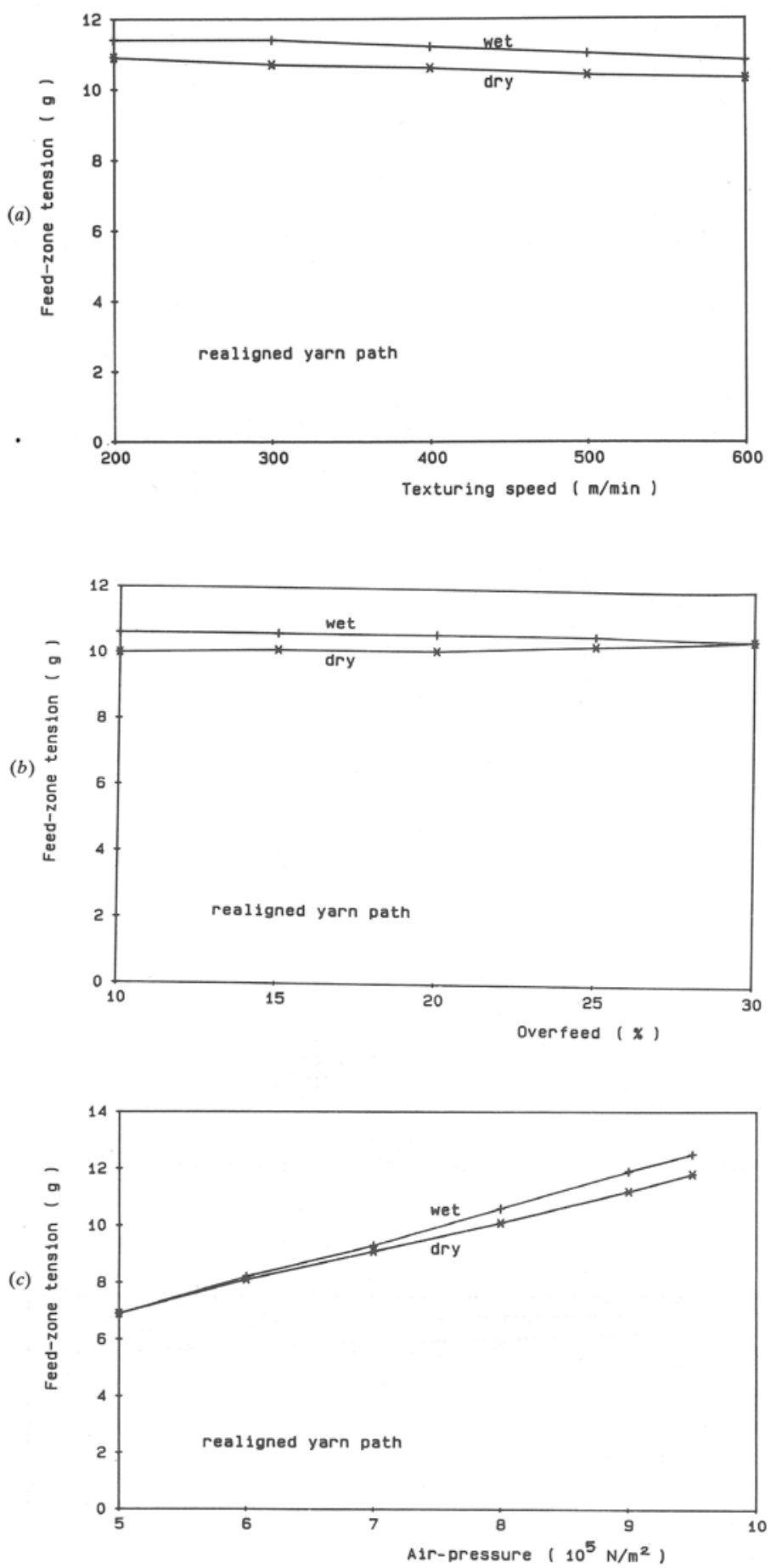

Fig. 4. Variation of tension in the feed zone with the realigned yarn path for changes in: (a) texturing speed; (b) overfeed ratio; and (c) air-pressure 

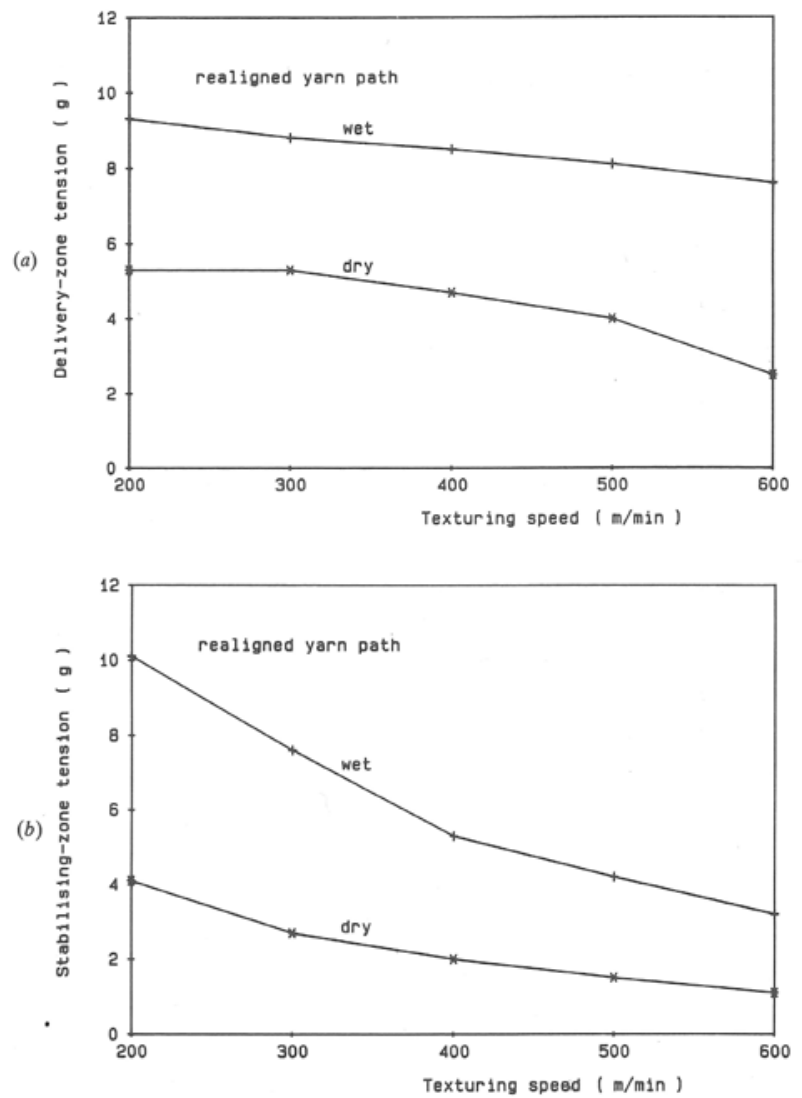

Fig. 5. The effects of texturing speed on tension in: (a) the delivery zone and (b) the stabilizing zone for the realigned yarn path
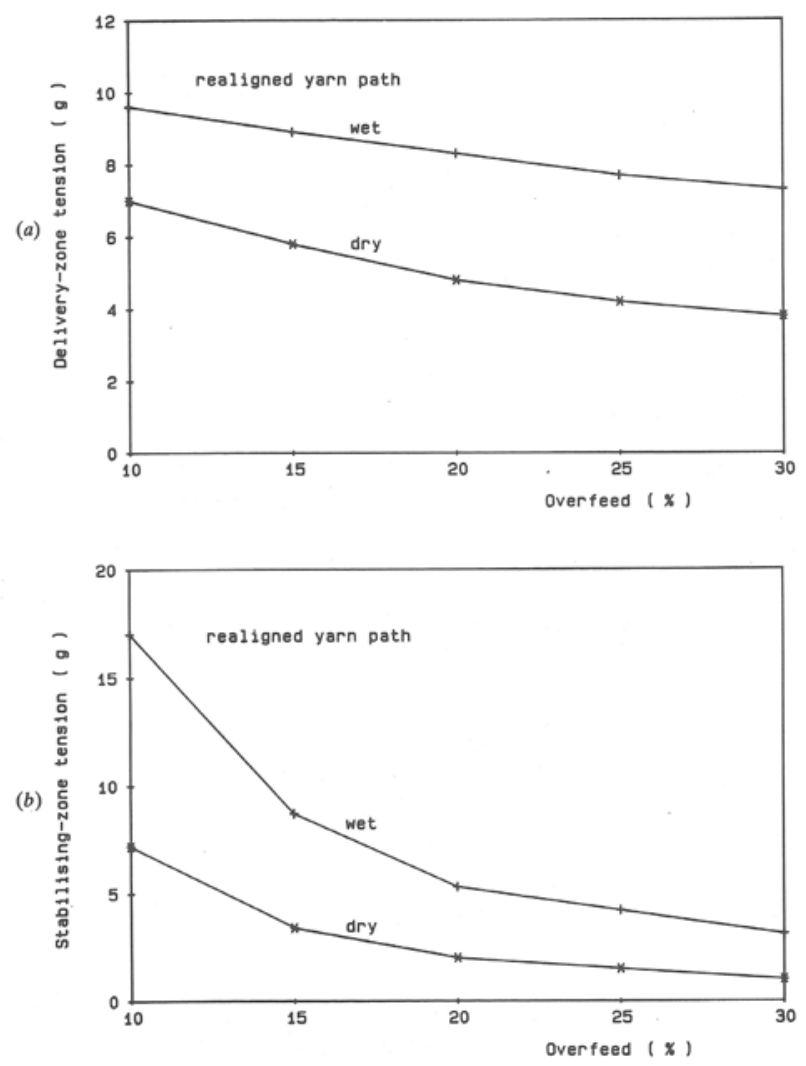

Fig. 6. The effects of overfeed variation on tension in: (a) the delivery zone and (b) the stabilizing zone for the realigned yarn path 

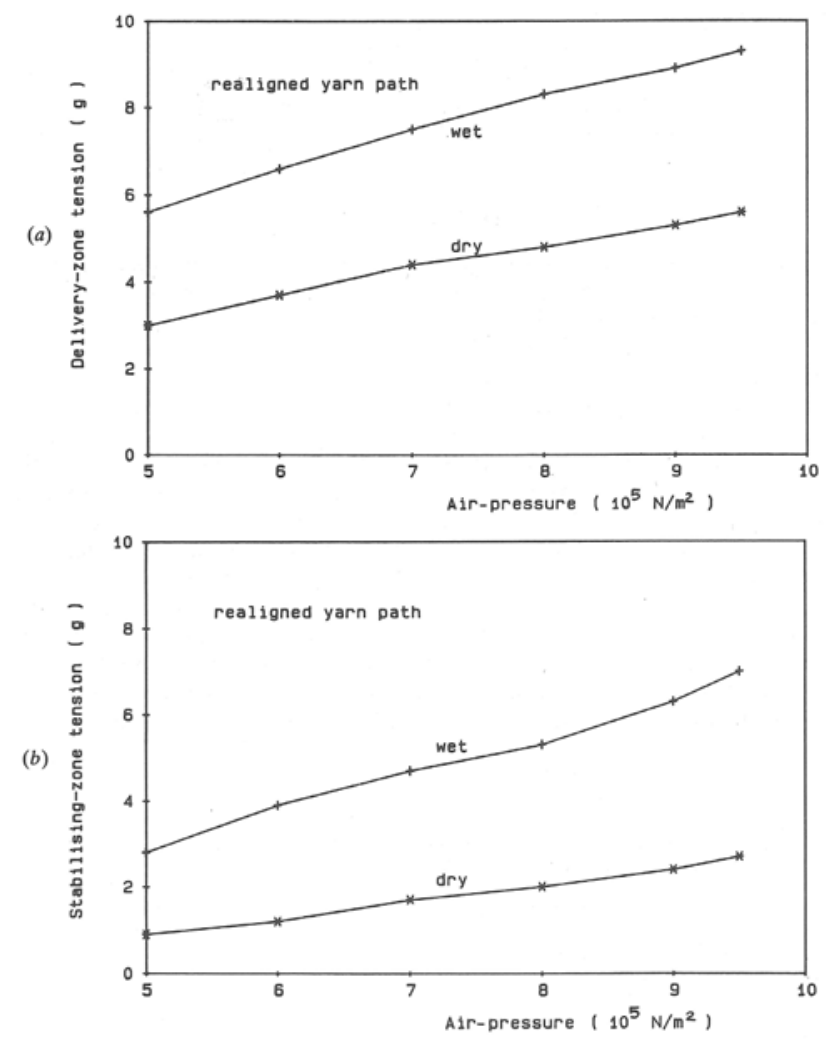

Fig. 7. The effects of air-pressure variation on tension in: (a) the delivery zone and (b) the stabilizing zone for realigned yarn path

\section{CONCLUSIONS}

The wetting of the filaments improves the effectiveness of the texturing process and results in better-quality textured yarns. Only a small amount of water is needed to impart the desired wetting effects. The water used in the wet-texturing process does not cause any significant changes in the flow velocity, which is the principal factor determining the fluid forces acting on the filaments.

Friction between the filaments themselves and friction between them and the contacting surfaces both play undesirable yet significant roles in the texturing process, because they reduce the resultant net forces acting on the filaments. The results show that wetting of the yarn in the air-jet texturing process produces a lubrication effect to reduce the friction between the filaments and the contacting parts, such as the texturing nozzle, the wetting unit, and the associated yarn guides. This causes a decrease in frictional forces and consequently an increase in the resultant forces acting on the filaments, which in turn results in yarns with improved properties. A similar reduction in friction can be obtained by realigning the feed roller, wetting unit, and nozzle.Moreover, wetting also reduces the friction between the filaments themselves, which enhances their longitudinal displacements relative to each other, facilitates better loop and entanglement formation, and thus generates improved textured yarns. 


\section{REFERENCES}

1. M. Acar, R. K. Turton, and G. R. Wray. J. Text. Inst., 1986, 77, 247.

2. M. Acar, R. TK. Turton, and G. R. Wray. J. Text. Isn't, 1986, 77, 235.

3. M. Acar and G. R. Wray. J. Text. Inst., 1986, 77, 377.

4. K. Fischer. Int. Text. Bull., Spinning, 1979, 17.

5. G. Bock and J. Luenenschloss in 'Textile Machinery: Investing for the Future', the Textile Institute, Manchester, 1982:

6. H. Artunc. Chemiefasern/ Textilindustrie, 1981, 31/83, 289 (E29).

7. W. W. Hansen and D. Tabor. Text. Res. J, 1957, 27, 300.

8. J. S. Olsen. Text. Res. J, 1969, 39, 31.

9. M. J. Schick. Text. Res. J, 1973, 43, 103.

10. M. J. Schick. Text. Res. J, 1973, 43, 342.

11. M. J. Schick. Text. Res. J, 1973, 43, 254.

12. M. J. Schick. 'Surface Characteristics of Fibres and Textiles, Part I', Dekker, New York and Basie, 1975.

13. G. R. Wray in 'Bulk, Stretch, and Texture' (edited by P. W. Harrison), the Textile Institute, Manchester, 1966, p. 18.

14. M. Acar, R. K. Turton, and G. R. Wray. J, Text. Inst., 1986, 77, 371. 